\title{
CAPACIDADE ADAPTATIVA DE ESPÉCIES DO CERRADO UTILIZADAS EM ÁREAS DE RECUPERAÇÃO AMBIENTAL ${ }^{1}$
}

Ana Carolina Ferreira Martins ${ }^{2}$, Ivan Schiavini ${ }^{3}$, Glein Monteiro de Araújo ${ }^{3}$ e Sérgio de Faria Lopes $^{4}$

\begin{abstract}
RESUMO - O objetivo deste estudo foi avaliar a capacidade adaptativa, com base em dados de área foliar específica (AFE) e comprimento do pecíolo (CP) de espécies nativas do Cerrado de duas áreas em recuperação, comparadas com indivíduos-controle. Foram selecionadas cinco espécies na Área 1 (luminosidade intensa) e 10 espécies na Área 2 (luminosidade intensa). Houve diferenças significativas entre as espécies das duas áreas e o controle, para a maioria das espécies estudadas. A AFE e o CP, ao contrário do que se esperava, foram significativamente maiores na Área 2 do que no controle (sombreamento). Entretanto, grande parte das espécies da Área 1 tiveram AFE e o CP iguais ou menores do que o controle e do que as espécies da Área 2. As espécies estudadas possuem, portanto, a capacidade de ajustar sua morfologia e, provavelmente, também sua fisiologia, para a aclimatação a ambientes com luminosidade intensa. Assim, são espécies potenciais para utilização em programas de recuperação ambiental.
\end{abstract}

Palavras-chave: Áreas degradadas; Área foliar específica; Comprimento do pecíolo.

\section{ADAPTATIVE CAPACITY OF CERRADO'S SPECIES USED IN ENVIRONMENTAL RECOVERY AREAS}

\begin{abstract}
The purpose of this study was to measure the adaptive capacity, based on data from specific leaf area (SLA) and petiole length (PL) of Cerrado's native species of two areas in recovery compared with control subjects. 5 species were selected in Area 1 (intense luminosity) and 10 species were selected in Area 2 (intense luminosity). There were significant differences between species of the two areas and the control subjects, for the most species evaluated. The SLA and PL, unlike what was expected, were significantly higher in Area 2 than control (shading). However, the most species in Area 1 had SLA and PL equal to or less than control and species of Area 2. Therefore, the studied species have ability to adjust its morphology and probably also physiology for acclimatization to the environments with intense luminosity. Thus, these are potential species for use in environmental recovery programs.
\end{abstract}

Keywords: Degraded areas; Specific leaf area; Pentiole Length.

\section{INTRODUÇÃO}

Nos últimos anos, novas formas de reflorestamento foram testadas, mudando também a forma de lidar com paisagens degradadas em florestas tropicais (LAMB et al., 2005). Em ambientes onde há ausência de regeneração natural, o plantio de mudas nativas no local é uma estratégia predominante para acelerar o processo de sucessão florestal (ISERNHAGEN et al., 2009; LAMB et al., 2005; CHAZDON, 2008). Para o sucesso no estabelecimento dessas plantas, é necessária a compreensão das relações entre variáveis ambientais e a vegetação (FERNANDES, 1998).

\footnotetext{
${ }^{1}$ Recebido em 11.04.2013 aceito para publicação em 07.04.2015.

${ }^{2}$ Universidade Federal de Uberlândia, Mestrado em Biologia Vegetal, Uberlândia, MG, Brasil. E-mail:<carolinamartins@ymail.com>.

${ }^{3}$ Universidade Federal de Uberlândia, Instituto de Biologia, Uberlândia, MG, Brasil. E-mail: <ivanschiavini@gmail.com> e<glein@ufu.br>.

${ }^{4}$ Universidade Estadual da Paraíba, Departamento de Biologia Geral, Instituto de Ciências Biológicas, Campina Grande, PB

- Brasil. E-mail: <defarialopes@gmail.com>.
} 
Entre todos os fatores ambientais que afetam o desenvolvimento e adaptação das plantas, principalmente em florestas tropicais, a luz é provavelmente o recurso de maior variação espacial e temporal, tornando-se fator limitante em muitos ambientes (PEARCY, 2007). A adaptação de espécies às condições heterogêneas de luminosidade pode levar a diversos padrões de plasticidade (SULTAN, 2003). As modificações morfológicas, fisiológicas e anatômicas que condicionam mudanças na estrutura e funções dessas plantas são importantes, sobretudo, na adaptação de mudas às condições de luz, pois determinam o sucesso ou não da regeneração (MACIEL et al., 2002).

A folha, por possuir todo o aparato fotossintético da planta, é o órgão que mais responde, de forma plástica, à variabilidade luminosa de determinado hábitat (CASTRO et al., 2005; CHIAMOLERA et al., 2010). Estratégias diferentes, em relação à morfologia foliar, são esperadas para plantas em condições de sombra e sob intensa luminosidade, quanto a uma melhor eficiência na captação de luz.

Plantas de ambientes mais sombreados tendem a ter folhas delgadas, área foliar específica (razão entre a área foliar e a massa seca da folha) e tamanho do pecíolo maiores, pois essas características ampliam a capacidade da captação de luz por unidade de biomassa foliar (NIINIMETS; FLECKS, 2002; DAHLGREN et al., 2006; LARCHER; BOERGER, 2009; POORTER; GARNIER, 2007). Essa é uma estratégia geralmente utilizada pelas plantas de sombra para se ter maior sucesso na competição pela luz (POORTER; GARNIER, 2007). Já as plantas de ambientes com maior disponibilidade de luz tendem a ter folhas mais espessas, por isso área foliar específica menor e tamanho do pecíolo também menor, pois a luz não é fator limitante nesse caso (REICH et al., 1997; EVANS; POOTER, 2001; REICH et al., 2003; LARCHER; BOERGER, 2009). $\mathrm{O}$ aumento na espessura da folha em espécies de luz acontece devido à maior quantidade de células que formam a camada paliçádica, protegendo a folha contra possíveis danos causados pela intensa luminosidade a que essas plantas ficam expostas (POOTER; GARNIER, 2007). A menor área foliar dessas espécies representa menor superfície exposta ao sol, diminuindo as taxas de transpiração e temperatura, não comprometendo a fotossíntese (NIINEMETS; FLECK, 2002). Espécies de locais mais secos também possuem tendência em apresentar área foliar específica menor do que espécies de áreas mais úmidas (POOTER; GARNIER, 2007), representando estratégia da planta contra a dessecação (ACKERLEY et al., 2002).

Neste estudo, objetivou-se avaliar a capacidade adaptativa de espécies de duas áreas em recuperação, comparadas com indivíduos-controle, ou seja, indivíduos das mesmas espécies em áreas de vegetação original do Cerrado, procurando responder às seguintes questões: a) as respostas morfológicas foliares apresentadas pelas espécies das duas áreas em recuperação diferem entre si e do controle? b) Por estarem sob radiação intensa e em diferentes condições ambientais, as espécies das áreas em recuperação apresentam traços foliares funcionais (área foliar específica e comprimento do pecíolo) menores do que o controle, que se encontra em maiores condições de sombreamento?

\section{MATERIAL E MÉTODOS}

\subsection{Locais de estudo}

As áreas em recuperação foram escolhidas com base no desenvolvimento das mudas, plantas que estavam com altura acima de $1 \mathrm{~m}$ e quantidade de folhas suficientes para a coleta. Além disso, são locais em que os fatores edáficos, a luminosidade e a inclinação do terreno são muito distintos, possibilitando comparar as mesmas espécies sujeitas a diferentes condições ambientais.

Área 1 - Compreende a Estação de Tratamento de Esgoto (ETE) Uberabinha, que está instalada no Município de Uberlândia, à margem direita do rio Uberabinha - corpo receptor do esgoto municipal tratado e principal fonte de abastecimento do Município de Uberlândia - próximo (a montante) à confluência com o córrego do Salto, situada à Estrada da Cachoeira, $\mathrm{s} / \mathrm{n}$, Fazenda do Salto, Distrito Industrial, com as coordenadas geográficas 1852'47.77"S e 48 19'44.07'O. Na ETE Uberabinha, a fisionomia predominante é a mata ciliar. É uma área de encosta com pouca umidade, já que o terreno é íngreme e a água escoa para o rio, não sendo retida no solo. As mudas encontravam-se em condições de luminosidade intensa, pois a área está em processo de regeneração, não havendo cobertura arbórea no local.

Área 2 - Representa uma das áreas de APP da Fazenda Santa Rosa. A fazenda está localizada na Bacia do Rio Uberabinha, no Município de Uberlândia, MG,

Revista Árvore, Viçosa-MG, v.39, n.3, p.543-550, 2015 
com as seguintes coordenadas $19^{\circ} 06^{\prime} 26.85^{\prime \prime} \mathrm{S}$ e $48^{\circ} 08^{\prime} 04.43^{\prime \prime} \mathrm{O}$. O local do plantio corresponde a uma mata de galeria inundável, apresentando-se praticamente plano. O solo é bastante úmido, evidenciando a drenagem deficiente do local. As mudas nessa área também se encontram sob radiação solar intensa, pelos mesmos motivos já apresentados na Área 1.

Controle - Inclui as coletas realizadas em locais de ocorrência natural das espécies estudadas. Como as espécies não foram encontradas em um único local, a coleta foi feita em pontos distintos, próximos à cidade de Uberlândia, MG (18 $55^{\circ} 08^{\prime \prime} \mathrm{S}$ e 48¹6’37" O). No entanto, todas as espécies estavam sob condições de sombreamento, pois os locais de coleta apresentavam vegetação fechada.

\subsection{Seleção das espécies}

Os critérios de seleção dos indivíduos, dentro das espécies estudadas, seguiram o protocolo elaborado por Cornelissen et al. (2003), sendo selecionados cinco indivíduos com características vigorosas, localizados em ambientes bem iluminados. Plantas que foram atacadas por herbívoros ou patógenos foram excluídas da seleção. Os indivíduos das Áreas 1 e 2 são indivíduos jovens, e os indivíduos utilizados como controle são adultos. $\mathrm{Na}$ Área 1 foram selecionadas para o estudo as seguintes espécies: Croton urucurana, Guazuma ulmifolia, Inga laurina, Inga sessilis e Tabebuia roseo-alba, totalizando cinco espécies. Na Área 2, além das espécies já citadas, Cecropia pachystachya, Luehea divaricata, Mysine umbellata, Tapirira guianensis e Trema micrantha também foram selecionadas, totalizando 10 espécies. Essas espécies representam, portanto, as espécies selecionadas para o controle.

\subsection{Coleta de dados}

O material para análise dos dados foi coletado já no final da estação chuvosa, nos meses de fevereiro, março e abril de 2012, e os métodos de coleta seguiram o protocolo estabelecido por Cornelissen et al. (2003).

Para cada indivíduo (cinco por espécie), foram coletadas de 5 a 10 folhas adultas totalmente expandidas (para minimizar a interferência do estádio de desenvolvimento foliar sobre seu tamanho). As folhas foram coletadas do $4^{\circ}$ nó, contado a partir da extremidade do ramo. O material coletado foi acondicionado em sacos plásticos fechados, para que não perdessem água até serem levados para o laboratório. Todos os indivíduos das Áreas 1 e 2 que tiveram suas folhas coletadas foram marcados com fita adesiva, para facilitar sua identificação, não coletando suas folhas mais de uma vez.

\section{4. Área foliar específica (AFE)}

As áreas foliares foram calculadas através de imagens digitalizadas das folhas de cada indivíduo (folhas completas), juntamente com uma escala métrica. Essas imagens foram obtidas através de um escâner de mesa, conectado a um computador e utilizando o programa especializado em análise de imagens, Image J (RASBAND, 1997). Aárea foliar das espécies de folhas compostas foi calculada considerando a folha como um todo e não apenas os folíolos. Em seguida, as folhas foram colocadas em estufa a $70^{\circ} \mathrm{C}$, por um período mínimo de 48 h e pesadas em balança de precisão, para obtenção da massa seca foliar. A área foliar específica foi mensurada através da razão área foliar $\left(\mathrm{mm}^{2}\right) /$ massa seca $(\mathrm{mg})$ para cada uma das folhas dos indivíduos coletados. Assim, a média da AFE de cada espécie foi obtida.

\subsection{Comprimento do pecíolo (CP)}

As mesmas imagens da obtenção da área foliar e do mesmo programa (Image J) foram utilizadas para o cálculo do comprimento do pecíolo de cada espécie estudada. Para as folhas compostas, foi estimado somente o pecíolo, desconsiderando-se o comprimento da raque foliar. Com a obtenção do valor do pecíolo de cada folha dos indivíduos selecionados, foi possível calcular a média do comprimento do pecíolo de cada espécie.

\subsection{Análise dos dados}

Para a comparação das médias dos traços foliares (AFE e CP) entre os indivíduos da mesma espécie selecionados nas Áreas 1,2 e controle, foi feita análise de variância (ANOVA). O teste T de Student foi feito para as comparações de média dos traços foliares da Área 2 com o controle. Esses testes foram aplicados a partir dos pressupostos de normalidade e homogeneidade dos dados. Nas comparações dos parâmetros que não atenderam a esses pressupostos, foram aplicados os testes não paramétricos de KruskalWallis e Mann-Whitney. A diferença entre as médias foi feita através do teste de comparações múltiplas

Revista Árvore, Viçosa-MG, v.39, n.3, p.543-550, 2015 
de Tukey a 5\% de significância. Para essas análises estatísticas, utilizou-se o programa SPSS (versão 16).

O cálculo do coefíciente de variação (CV), definido como o desvio-padrão em porcentagem da média, que é também a medida estatística mais utilizada pelos pesquisadores na avaliação da precisão dos experimentos (AMARAL et al., 1997), foi usado para medir a variabilidade intraespecífica entre as amostras de uma mesma espécie.

\section{RESULTADOS}

As médias dos valores da área foliar específica (AFE), comprimento do pecíolo (CP) e o coeficiente de variação $(\mathrm{CV})$ das espécies selecionadas nas Áreas 1,2 e controle estão na Tabela 1 .

\section{1. Área foliar específica (AFE)}

Os valores da AFE, obtidos nas Áreas 1, 2 e controle, indicam que a Área 1 obteve o menor índice, com exceção de Guazuma ulmifolia, que apresentou maior AFE nessa área do que no controle (Tabela 1). Já na Área 2 as espécies demonstram tendência em apresentar valores maiores de AFE, comparados aos da Área 1 e do controle.
Tudo isso com exceção de Croton urucurana, que obteve valor intermediário entre a Área 1 e o controle (Tabela 1).

O CV das cinco espécies comparadas nas Áreas 1,2 e no controle foi inferior a $20 \%$. Isso demonstra que a variabilidade dos dados não influenciou, de maneira negativa, nos resultados, sendo as amostras de boa precisão (PIMENTEL-GOMES, 1985).

Para os resultados entre a Área 2 e o controle, Tapirira guianensis e Trema micrantha não obtiveram médias significativamente diferentes da AFE. Contudo, as demais espécies apresentaram variações significativas entre as médias da AFE. No entanto, Luehea divaricata, Tapirira guianensis e Trema micrantha apresentaram CV maior que $20 \%$, as duas primeiras no controle e a última na Área 2. Esses valores apresentam baixa precisão em relação aos resultados obtidos com as amostras que foram coletadas, o que limita o alcance das comparações (PIMENTEL-GOMES, 1985). Isso pode ter ocorrido devido ao fato de a época de coleta, que foi feita já no final da estação chuvosa, e muitas folhas não se encontravam em boas condições para serem coletadas, principalmente no controle por se tratar de plantas adultas.

Tabela 1 = Médias da área foliar específica e do tamanho do pecíolo de cada espécie selecionada nas Áreas 1 e 2, comparadas ao controle. $\mathrm{AFE}=$ média de área foliar específica $\left(\mathrm{mm}^{2} / \mathrm{mg}\right) ; \mathrm{A} 1=$ Área 1 (ETE); A2 = Área 2 (Fazenda Santa Rosa); $\mathrm{C}=$ controle; e $\mathrm{CP}=$ média do comprimento do pecíolo $(\mathrm{cm}) . \mathrm{CV}=$ Coeficiente de variação das amostras de cada espécie em cada área em que foi coletada. Letras diferentes nas linhas indicam diferenças estatisticamente significativas entre as médias a 5\% de probabilidade, pelo teste de Tukey, e * indica diferença significativa entre as médias da Área 2 e as médias do controle a 5\% de probabilidade.

Table 1 - Averages of the specific leaf area and petiole length of each species selected in Areas 1 and 2 compared to control. $S L A=$ Average of the specific leaf area $\left(\mathrm{mm}^{2} / \mathrm{mg}\right) ;$ A1 = Area 1 (ETE), A2 = Area 2 (Santa Rosa Farm), C = control, $P L=$ average of the petiole length (cm). $C V=$ coefficient of variation of the samples of each species in each area in which it was collected. Different letters in rows indicate significant differences between means at $5 \%$ probability by Tukey test and * indicates a significant difference between the averages of Area 2 and the means of control at $5 \%$ probability.

\begin{tabular}{|c|c|c|c|c|c|c|c|c|c|}
\hline \multirow{2}{*}{ Espécies } & \multicolumn{3}{|c|}{ AFE } & \multicolumn{3}{|c|}{$\mathrm{CP}$} & \multicolumn{3}{|c|}{$\mathrm{CV}$} \\
\hline & A1 & $\mathrm{A} 2$ & $\mathrm{C}$ & A1 & $\mathrm{A} 2$ & $\mathrm{C}$ & A1 & $\mathrm{A} 2$ & $\mathrm{C}$ \\
\hline Cecropia pachystachya Trécul & & $10,05 *$ & $7,81 *$ & - & $13,18 *$ & $21,98 *$ & - & $14,87 \%$ & $10,88 \%$ \\
\hline Croton urucurana Baill. & $12,20 \mathrm{a}$ & $13,08 \mathrm{ab}$ & $13,76 \mathrm{~b}$ & $7,47 \mathrm{~b}$ & $5,24 \mathrm{a}$ & $9,18 \mathrm{c}$ & $18,35 \%$ & $14,60 \%$ & $12,48 \%$ \\
\hline Guazuma ulmifolia Lam. & $11,56 \mathrm{~b}$ & $12,87 \mathrm{c}$ & $8,72 \mathrm{a}$ & $0,94 \mathrm{a}$ & $1,13 \mathrm{~b}$ & $1,22 \mathrm{~b}$ & $3,90 \%$ & $11,29 \%$ & $12,80 \%$ \\
\hline Ingalaurina (Sw.) Willd. & $6,56 \mathrm{a}$ & $7,43 \mathrm{~b}$ & $7,43 \mathrm{~b}$ & $1,2 \mathrm{a}$ & $1,46 \mathrm{~b}$ & $1,46 \mathrm{~b}$ & $15,62 \%$ & $8,98 \%$ & $11,22 \%$ \\
\hline Inga laurina (Vell.) Mart. & $8,00 \mathrm{a}$ & $10,24 b$ & $8,65 \mathrm{a}$ & $1,11 \mathrm{a}$ & $1,71 \mathrm{~b}$ & $1,76 \mathrm{~b}$ & $14,13 \%$ & $15,67 \%$ & $19,14 \%$ \\
\hline Luehea divaricata Mart. & - & $10,34 *$ & $14,61 *$ & - & $0,93 *$ & $0,84 *$ & - & $16,97 \%$ & $31,24 \%$ \\
\hline Mysine umbellata Mart & - & $8,86^{*}$ & $6,65^{*}$ & - & $1,11^{*}$ & $1,31^{*}$ & - & $19,83 \%$ & $12,29 \%$ \\
\hline Tabebuia roseo-alba (Ridl.) Sand. & $8,76 \mathrm{a}$ & $9,78 \mathrm{~b}$ & $9,18 \mathrm{ab}$ & $7,04 \mathrm{a}$ & 8,09 a & 7,98 a & $12,62 \%$ & $6,19 \%$ & $13,88 \%$ \\
\hline Tapirira guianensis Aubl. & - & 7,80 & 7,43 & - & $5,56^{*}$ & $3,92 *$ & - & $17,15 \%$ & $24,47 \%$ \\
\hline Trema micrantha (L.) Blum. & - & 13,95 & 13,84 & - & $0,85^{*}$ & $1,07 *$ & - & $26,26 \%$ & $19,35 \%$ \\
\hline
\end{tabular}

Revista Árvore, Viçosa-MG, v.39, n.3, p.543-550, 2015 
Cecropia pachystachya e Myrsine umbellata, cujo CV foi inferior a $20 \%$, tiveram comportamento semelhante ao das espécies comparadas nas Áreas 1, 2 e controle, ou seja, o valor da AFE foi significativamente maior na Área 2 do que no controle. Portanto, para a análise das respostas morfológicas foliares, as espécies apresentaram valores de médias significativamente diferentes entre si e diferentes do controle, na maioria das espécies. As espécies estudadas somente na Área 2 também diferiram do controle, em sua maioria.

\subsection{Pecíolo}

Para os valores de CP, também houve diferença significativa entre as médias das Áreas 1, 2 e o controle de todas as espécies, com exceção de Tabebuia roseoalba, que não apresentou diferença entre as médias relatadas. Assim como ocorreu nos valores médios da AFE, a Área 1 também apresentou os menores valores no comprimento do pecíolo, a não ser por Croton urucurana, que obteve média maior nessa área do que na Área 2. Para essa análise, a situação foi um pouco diferente do que aconteceu com a AFE, em que a Área 2 apresentou os maiores valores, pois, com exceção de Crotonurucurana, não houve diferença significativa entre os valores médios do comprimento do pecíolo entre a Área 2 e o controle.

Já na análise das espécies estudadas somente na Área 2, comparadas ao controle, o CP foi consideravelmente maior no controle para a maioria das espécies, principalmente em Cecropia pachystachya. No entanto, Luehea divaricata e Tapirira guianensis, que foram as espécies que apresentaram valores menores no controle do que na Área 2, tiveram o CV superior a $20 \%$, como já comentado anteriormente.

Para essa análise, portanto, a diferença do CP ocorreu entre as espécies das duas áreas, mas não entre a Área 2 e o controle, para a maioria das espécies, sendo menores os valores na Área 1. Já nas espécies estudadas apenas na Área 2 a diferença foi significativa entre todas as espécies dessa área e do controle.

\section{DISCUSSÃO}

Pode-se inferir que os atributos foliares funcionais (AFFs) interferem na habilidade das plantas em adquirir, usar e conservar recursos, sendo, portanto, decisivos nas estratégias de investimento e crescimento que elas possuem (WRIGHT et al., 2004). Para a análise desses atributos, era esperado que os valores da AFE fossem significativamente maiores no controle, devido à condição de sombreamento a que as espécies se encontravam, já que muitos estudos demonstraram que a AFE e o comprimento do pecíolo são maiores em ambientes sombreados (LICHTENTHALER et al., 1981; NIINIMETS; FLECKS, 2002; LIMA-JÚNIOR etal., 2005; DAHLGREN et al., 2006; POOTER; GARNIER, 2007; LARCHER; BOERGER, 2009). No entanto, na Área 1 foram obtidos valores iguais ou inferiores aos do controle e, na Área 2 , as espécies tiveram tendência em apresentar valores significativamente maiores do que os do controle, mesmo para aquelas espécies estudadas somente nessa última área (com coeficiente de variação superior a 20\%). Isso, provavelmente, aconteceu devido à variabilidade nesses traços funcionais que ocorre entre e dentro das espécies, em nível de indivíduos e até entre folhas de um mesmo indivíduo, decorrente de fatores ambientais, genéticos e do estágio de desenvolvimento desses indivíduos (COLEMAN et al., 1994; BRUSCHI et al., 2003; ALBERT et al., 2010; ALBERT et al., 2011). Além disso, não é possível inferir a respeito da procedência genética dessas espécies, ou seja, se os indivíduos são de uma mesma matriz ou de matrizes diferentes, já que foram plantas adquiridas em diversos viveiros.

Da mesma forma, esperava-se que o comprimento do pecíolo fosse maior no controle, devido à necessidade das plantas em aumentar a área para a interceptação de luz. No entanto, a Área 2 obteve valores significativamente iguais aos do controle e maiores do que a Área 1. Isso provavelmente ocorreu porque, como houve tendência de as espécies nessa área terem maior área foliar, o CP teve que ser proporcional a essa variação. Mas o comprimento menor do pecíolo em espécies com maiores valores de AFE também apareceu em determinadas espécies (Cecropia pachystachya, Luehea divaricata, Mysine umbellata). Entretanto, nenhuma correlação foi feita para avaliar com precisão essa hipótese. Já a maioria das espécies da Área 1 comportou-se dentro do esperado para essa variável, pois obteve valores significativamente menores, em comparação com o controle.

Muitos estudos demonstram que características foliares como o tamanho da folha e comprimento do pecíolo alteram a eficiência da folha na captação de luz (NIINEMETS et al., 2004). Mas o pecíolo, além de ser estratégia da planta para expor, de forma eficiente, a lâmina foliar à luz também funciona como suporte

Revista Árvore, Viçosa-MG, v.39, n.3, p.543-550, 2015 
mecânico que sustenta a folha (NIINEMETS; FLECK, 2002). Por isso, a relação entre comprimento do pecíolo e massa foliar varia de acordo com a geometria do pecíolo e o formato da folha (NIKLAS, 1991; NIINEMETS et al., 2004).

As diferenças obtidas podem ser indicativas da amplitude ecológica dessas espécies. A variação encontrada entre comparações significa que as características avaliadas desenvolveram capacidade plástica (SCHLICHTING, 1986). Essas espécies podem colonizar uma variedade de hábitats, porque apresentam alta plasticidade fenotípica em relação a ambientes com diferentes regimes de luz (ROZENDAAL et al., 2006).

Já as espécies que não apresentaram diferença significativa podem ser consideradas como adaptadas ao ambiente em regeneração. A adaptação é o resultado da variabilidade funcional a uma série de fatores e que foi passada aos seus descendentes, por isso respostas adaptativas estão fixadas ao genótipo, e variações ambientais não modificam o fenótipo (SCHLICHTING, 1986; REICH et al., 2003; AOYAMA; MAZZONIVIVEIROS, 2006). O sucesso na adaptação de uma espécie em diferentes condições de radiação pode ser baseado na eficácia e rapidez que os padrões de alocação de biomassa e comportamento fisiológico são ajustados (ALMEIDA et al., 2004; LIMA JÚNIOR et al., 2005). A alocação de biomassa pelas folhas que ocorre de maneira desigual em indivíduos de uma mesma espécie que cresce sob condições de luminosidade diferente é uma evidência dessa adaptação (COLEMAN et al., 1994).

De acordo com Reich et al. (2003), a sobrevivência à colonização de um novo ambiente por uma espécie ou alterações nesse ambiente só será possível se seus traços funcionais forem adequados a esse ambiente. Vários estudos apontam que espécies de crescimento rápido, consideradas como pioneiras, são favorecidas por ambientes de luz e parecem exibir maior plasticidade em comparação com espécies de crescimento lento e tolerantes à sombra (ROZENDAAL et al., 2006; POOTER; GARNIER, 2007). Muitos estudos também têm demonstrado efeito positivo da disponibilidade de luz na fisiologia e desempenho do crescimento em mudas de espécies arbóreas (POOTER; GARNIER, 2007).

A AFE está positivamente correlacionada com a capacidade fotossintética máxima da folha e, em consequência disso, está também associada ao crescimento da planta (CORNELISSEN et al., 2003). Por isso, para determinadas plantas em estágio de desenvolvimento, a taxa de crescimento é maior sob um nível maior de irradiância, assim como tendência no favorecimento do crescimento da raiz e maiores taxas de assimilação líquida de carbono (OSUNKOYA et al., 1994; ILLENSEER; PAULILO, 2002). Assim, o gasto energético com uma AFE maior em espécies sob maior irradiância seria justificado pela necessidade dessas plantas em crescer nesses ambientes.

\section{CONCLUSÃO}

As respostas apresentadas por essas espécies diferiram, em grande parte, entre as áreas estudadas e em comparação com o controle, apresentando traços foliares funcionais maiores do que esse, o que não era esperado para plantas sob regime de luz intensa. Esse comportamento pode ser considerado uma capacidade plástica dessas plantas. Já naquelas espécies que não tiveram diferença significativa em relação ao ajustamento morfológico nas áreas estudadas, o comportamento adaptativo delas foi a estratégia que garantiu o sucesso dessas plantas nesses ambientes. Entretanto, é importante levar em consideração que as espécies aqui estudadas foram coletadas na fase jovem e, por isso, são mais vulneráveis a fatores abióticos e bióticos.

\section{AGRADECIMENTOS}

À CAPES, pela bolsa de mestrado concedida ao primeiro autor; e à Gerência Ambiental do Departamento Municipal de Água e Esgoto de Uberlândia (DMAE), em especial a Priscila Franco e Cairo Silva, pelo apoio.

\section{REFERÊNCIAS}

ACKERLEY, D.; KNIGHT, C.; WEISS, S.; BARTON, K.; STARMER, K. Leaf size, specific leaf area and microhabitat distribution of chaparral woody plants: contrasting patterns in species level and community level analyses. Oecologia, v.130, n.3, p. 449-457, 2002.

ALBERT, C.H.; GRASSEIN, F.; SCHURR, F.M.; VIEILLEDENT, G.; VIOLLE, C. When and how should intraspecific variability be considered in trait-based plant ecology? Perspectives in Plant Ecology, Evolution and Systematics, v.13, p.217-225, 2011. 
ALBERT, C.H.; THUILLER, W.; YOCCOZ, N.G.; SOUDANT, A.; BOUCHER, F.; SACCONE, P.; LAVOREL, S. Intraspecific functional variability: extent, structure and sources of variation.

Journal of Ecology, v.98, n.3, p.604-613, 2010.

ALMEIDA, L.P.; ALVARENGA, A.A.; CASTRO, E.M.; ZANELA, S.M.; VIEIRA, C.V. Crescimento inicial de plantas de Cryptocaria aschersoniana Mez. submetidas a níveis de radiação solar.

Ciência Rural, v.34, n.1, p.83-88, 2004.

AMARAL, A.M.; MUNIZ, J.A.; SOUZA, M.

Avaliação do coeficiente de variação como medida da precisão na experimentação com citros.

Pesquisa Agropecuária Brasileira, v.44, p. 1221-1225, 1997.

AOYAMA, E.M.; MAZZONI-VIVEIROS, S.C. Adaptações estruturais das plantas ao ambiente. Curso de Capacitação de monitores e educadores. Instituto de Botânica, 2006 Disponível em: http://

www.biodiversidade.pgibt.ibot.sp.gov.br/Web/ PublicacoesDidaticas.aspx Acesso em dez. de 2012.

BRUSCHI, P.; GROSSONI, P.; BUSSOTI, F. Withinand among-tree variation in leaf morphology of Quercus petraea (Matt.) Liebl. natural populations. Trees, v.17, n.2, p.164-172, 2003.

CASTRO, E.M.; PINTO, J.E.B.P.; MELO, H.C.; SOARES, A.M.; ALVARENGA, A.A.; LIMA JUNIOR, E.C. Aspectos anatômicos e fisiológicos de plantas de guaco submetidas a diferentes fotoperíodos. Horticultura Brasileira, v. 23, n.3, p.846-850, 2005.

CHAZDON, R.L. Beyond deforestation: Restoring forests and ecosystem services on degraded lands. Science, v.320, p.1458-1460, 2008.

CHIAMOLERA, L.B.; ÂNGELO, A.C.; BOERGER, M.R. Resposta morfoanatômica da folha de Podocarpus lambertii Klotzsch ex Endl (Podocarpaceae) implantado em duas áreas com diferentes graus de sucessão às margens do Reservatório Iraí - PR. Revista Biotemas, v.23, n.1, p.1-11, 2010.

COLEMAN, J.S.; MCCONNAUGHAY, K.D.M.; ACKERLY, D.D. Interpreting phenotypic variation in plantas. Tree, v.8, n.5, p.187-191, 1994.
CORNELISSEN, J.H.C.; LAVOREL, C.S.; GARNIER, E.; DIAZ, S.; BUCHMANN, N.; GUVICH, D.E.; REICH, P.B.; TER STEEGE, H.; MORGAN, H.D.; van der HEIJDEN, M. G.A.; PAUSAS, J.G.; POOTER, H. Handbook of protocols for standardised and easy measurement of plant functional traits worldwide. Australian Journal of Botany, v.51, p.335-380, 2003.

DAHLGREN, J.P.; ERIKSSON, O.; BOLMGREN, K.; STRINDELL, M.; EHRLÉN, J. Specific leaf area as a superior predictor of changes in field layer abundance during forest succession. Journal of vegetation. Science, v.17, p.577-582, 2006.

EVANS, J.R.; POORTER, H. Photosynthetic acclimation of plants to growth irradiance: the relative importance of specific leaf area and nitrogen partitioning in maximizing carbon gain. Plant, Cell and Environment, v.24, p.755-767, 2001.

FERNANDES, H.A.C. Dinâmica e distribuição de espécies arbóreas em uma floresta secundária no domínio da Mata Atlântica. 1998. 148f. Dissertação (Mestrado em Engenharia Florestal). Universidade Federal de Viçosa, Viçosa, MG, 1998.

ILLENSEER, R.; PAULILO, M.T.S. Crescimento e eficiência na utilização de nutrientes em plantas jovens de Euterpe edulis Mart. Sob dois níveis de irradiância, nitrogênio e fósforo. Acta

Botânica Brasílica, v. 16, n.4, p.385-394, 2002.

ISERNHAGEN, I.; BRANCALION, P.H.S.; RODRIGUES, R.R.; NAVE, A.G. GANDOLFI, S. Diagnóstico ambiental das áreas a serem restauradas visando a definição de metodologias de restauração florestal. In: RODRIGUES, R.R.; BRANCALION, P.H.S.; ISERNHAGEN, I. (Eds.). Pacto pela restauração da Mata Atlântica: referencial dos conceitos e ações de restauração florestal. São Paulo: LERF/ ESALQ: Instituto BioAtlântica, 2009. p.87-127.

LAMB, D.; ERSKINE, P.D.; PARROTA, J.A. Restoration of degraded tropical forest landscapes. Science, v.310, p.1628-1632, 2005.

LARCHER, L.; BOEGER, M.R.T. Arquitetura foliar de Odontonema strictum (Ness) O. Kuntze (Acanthaceae) em duas condições de luminosidade. Hoehnea, v.36, p.321-327, 2009.

Revista Árvore, Viçosa-MG, v.39, n.3, p.543-550, 2015 
LICHTENTHALER, H.K.; BUSCHMANN, C.; DOLL, M.; FIETZ, H.J.; BACH, T.; KOZEL, U.; MEIER, D.; RAHMSDORF, U. Photosynthetic activity, chloroplast ultrastructure, and leaf characteristics of high-light and low-light plants and of sun and shade leaves. Photosynthesis Research, v.2, p.115-141, 1981.

LIMA JÚNIOR, E.C.; ALVARENGA, A.A.; CASTRO, E.M.; VIEIRA, C.V.; OLIVEIRA, H.M. Trocas gasosas, características das folhas e crescimento de plantas jovens de Cupania vernalis Camb. submetidas a diferentes níveis de sombreamento. Ciência Rural, v.35, p.1092-1097, 2005.

MACIEL, M.N.M.; WATZLAWICK, L.F.; SCHOENINGER, E.R.; YAMAJI, F.M. Efeito da radiação solar na dinâmica de uma floresta.

Revista Ciências Exatas e Naturais, v.4, n.1,p.101-114, 2002.

NIINEMETS, U.; FLECK, S. Petiole mechanics, leaf inclination, morphology, and investment in support in relation to light availability in the canopy of Liriodendrontu lipifera. Oecologia, v.132, p.21-33, 2002.

NIINEMETS, U.; AFAS, N.A.; CESCATTI, A.; PELLIS, A.; CEULEMANS, R. Petiole length and biomass investment in support modify lightinterception efficiency in dense popular plantations. Tree Physiology, v.24, p.141-154, 2004.

NIKLAS, K.J. Flexural stiffness allometries of angiosperm and fern petioles and rachises: evidence for biomechanical convergence. Evolution, v.45, p.734-750, 1991.

OSUNKOYA, O.O.; ASH, J.E.; HOPKINS, M.S.; GRAHAM, A.W. Influence of seed size and seedling ecological attributes on shade-tolerance of rain forest tree species northern Queesland. Journal of Ecology, v.82, n.1, p.149-163, 1994.

PEARCY, R.W. Responses of plants to heterogeneous light environments. In: PUGNAIRE, F.I.; VALLADARES F. (Eds.). Functional plant ecology. 2.ed. Boca Raton: CRC Press, 2007. p.213-246.

PIMENTEL-GOMES, F. Curso de estatística experimental, $11^{\mathrm{a}}$ ed. Piracicaba: Nobel, 1985. p.466.

Revista Árvore, Viçosa-MG, v.39, n.3, p.543-550, 2015
POORTER, H.; GARNIER, E. Ecological Significance of inherent variation in relative growth rate and its components. In: PUGNAIRE, F.I.; VALLADARES, F. (Eds.). Functional plant ecology. Boca Raton: CRC Press, 2007.p.67-100.

RASBAND, W.S. ImageJ, U. S. National Institutes of Health. Bethesda: 1997. Disponível em: http://imagej.nih.gov/ij/. Acesso em fev. de 2012.

REICH, P.B.; WALTERS, M.B.; ELLSWORTH, D.S. From tropics to tundra: Global convergence in plant functioning. Proceedings National Academic Science USA, v.94, p.13730$13734,1997$.

REICH, P.B.; WRIGHT, I.J.; BARES-CAVENDER, J.; CRAINE, J.M.; OLEKSYN, J.; WESTOBY, M. ; WALTERS, M.B. The evolution of plant functional variation: traits, spectra, and strategies.

International Journal of Plant Sciences, v.164, n.3, p.143-164. 2003.

ROZENDAAL, D.M.A.; HURTADO, V.H.; POORTER, L. Plasticity in leaf traits of 38 tropical tree species in response to light; relationships with light demand and adult stature. Functional Ecology, v.20, n.2, p.207-216, 2006.

SCHLICHTING, C.D. The evolution of phenotypic plasticity in plants. Annual

Review of Ecological and

Systematics, v.17, p.667-693, 1986.

SULTAN, S.E. Phenotypic plasticity in plants: a case study in ecological development. Evolution \& Development, v.5, n.1, p.25-33, 2003.

WRIGHT, I.J.; REICH, P.B.; WESTOBY, M.; ACKERLY, D.D.; BARUCH, Z.; BONGERS, F.; CAVENDER- BARES, J.; CHAPIN, T.; CORNELISSEN, J.H.C.; DIEMER, M.; FLEXAS, J.; GARNIER, E.; GROOM, P.K.; GULIAS, J.; HIKOSAKA, K.; LAMONT, B.B.; LEE, T.; LEE, W.; LUSK, C.; MIDGLEY, J.J.; NAVAS, M.L.; NIINEMETS, U.; OLEKSYN, J.; OSADA, N.; POORTER, H.; POOT, P.; PRIOR, L.; PYANKOV, V.I.; ROUMET, C.; THOMAS, S.C.; TJOELKER, M.G.; VENEKLAAS, E.J.; VILLAR, R. The worldwide leaf economics spectrum. Nature, v.428, p.821-827, 2004. 